Arq. Bras. Med. Vet. Zootec., v.64, n.2, p.458-464, 2012

\title{
Confiança e agregação de valor em carnes com indicação geográfica
}

\author{
[Trust and added value on meat with geographic indication] \\ F.S. Brandão, A.C. Ceolin, M.E.A. Canozzi, J.P.P. Révillion, J.O.J. Barcellos \\ Universidade Federal do Rio Grande do Sul - UFRGS - Porto Alegre, RS
}

\begin{abstract}
RESUMO
$\mathrm{O}$ aumento da procura por produtos agroalimentares com certificação relacionada à origem geográfica tem ocorrido, buscando atender nichos específicos de mercado. Nesse sentido, este trabalho identificou a percepção dos consumidores brasileiros com relação às indicações geográficas e sua disposição em pagar por esse atributo. Como método, realizou-se uma survey utilizando-se o software Sphinx, via internet, com 272 consumidores de carne bovina. Constatou-se que a percepção do consumidor sobre as indicações geográficas em carnes é, de maneira geral, positiva, sendo este atributo reconhecido como um indicador de qualidade. Os consumidores entrevistados acreditam que essas carnes oferecem maior segurança alimentar e são mais confiáveis que o produto sem a indicação de origem geográfica, sendo possível agregar valor em função de tais diferenciais. Além disso, o consumidor valorizou esse atributo e está disposto a pagar mais pelas carnes com selo de indicação geográfica.
\end{abstract}

Palavras-chave: carne bovina, certificação, diferenciação, consumidor, Brasil

\begin{abstract}
The increased demand for agrifood products with certification related to the geographical origin has occurred on both global and national levels, seeking to meet specific market niches. In this sense, this article aims to identify consumer perceptions with respect to geographical indicators and their willingness to pay for this attribute. As a method, there was an internet survey among 272 consumers of beef with the help of the Sphinx software. It was found that consumer perception on geographical indications in meat is generally positive, and this attribute is recognized as a quality indicator. Consumers believe that this meat offers security and is more reliable than the product that doesn't mention source, and you can add value to these differences. In addition, consumers value this attribute and are willing to pay more for meat with the seal of geographical indication.
\end{abstract}

Keywords: differentiation, consumer behavior, beef, certification, Brazil

\section{INTRODUÇÃO}

Ao longo das últimas décadas, os problemas vivenciados na produção animal - crises sanitárias em animais e doenças em humanos aumentaram a preocupação com a segurança do alimento (Sepúlveda et al., 2008). Ademais, a associação negativa entre o ambiente e a expansão da pecuária, sendo esta atividade responsabilizada pelo desmatamento e contribuinte na emissão de gases do efeito estufa na atmosfera, prejudica a imagem da carne bovina no cenário internacional (Nemecek et al., 2011). Assim, uma diversidade de produtos agroalimentares surge no final do século $\mathrm{XX}$, para atender às exigências da demanda. Contudo, essa variedade pode dificultar o processo de compra, uma vez que os consumidores raramente conseguem obter o total das informações que necessitariam para tomar uma decisão completamente desprovida de incerteza (Assael, 1992). Por essa razão, muitas vezes, os consumidores recorrem a inferências da realidade que são fortemente baseadas em suas percepções. Portanto, na impossibilidade de

Recebido em 21 de novembro de 2011

Aceito em 12 de janeiro de 2012

E-mail: ferbran@terra.com.br 
acesso a todas as informações e variáveis envolvidas no processo de decisão do consumidor, este passa a sofrer influência das informações extrínsecas ao produto, como as certificações e o país de origem (Guilhoto, 2001).

Com a globalização, o comércio de alimentos certificados desenvolve-se rapidamente, e os consumidores, em todo o mundo, apresentam um interesse crescente por alimentos com identidades locais e regionais garantidas (Font i Furnols et al., 2011; Menapace et al., 2011; Schnettler et al., 2011), sendo essas certificações apropriadas para assegurar maior qualidade e segurança (Zuin e Zuin, 2008).

A indicação geográfica (IG) aparece neste cenário de incertezas como uma verdadeira garantia para o consumidor, indicando que se trata de um produto genuíno, cuja especificidade se deve a sua origem (Rojas, 2004; Tápias, 2005), conferindo ao produto um diferencial de mercado. $\mathrm{O}$ vínculo gerado entre o produto e o território ocorre, segundo Vargas (2008), pela delimitação das zonas de produção, tecnologias de produção e elaboração, controle de qualidade e conhecimento, os quais garantem a especificidade da região.

A diferenciação dos produtos - fruto de ações coletivas locais por meio da criação de marcas geográficas como forma de valorizar usos e costumes locais - pode proporcionar um diferencial competitivo para empresas agroalimentares (Malafaia et al., 2011). Desse modo, a indicação de origem geográfica poderia ser considerada como um atributo diferenciador, pois, dependendo do contexto em que se enquadra, apresenta qualidades que não são comuns a todos os produtos. No entanto, o grau de conhecimento por parte do consumidor e a sua disposição em pagar um diferencial pela carne com IG ainda carece de estudos. Nesse sentido, este trabalho teve o objetivo de identificar o perfil do consumidor de carne e sua percepção e disposição para pagar pela carne com indicação geográfica.

\section{MATERIAL E MÉTODOS}

A pesquisa caracterizou-se como survey. A partir da revisão de literatura em periódicos nacionais e internacionais sobre comportamento do consumidor de carne bovina, foi elaborado um questionário semiestruturado composto por questões fechadas - únicas, múltiplas e escalares do tipo likert de cinco pontos - e abertas questões "outro e justifique". Esse foi subdividido em duas dimensões: a primeira sobre o perfil do consumidor de carne bovina, e a segunda referente às características da $\mathrm{IG}$ em carnes - qualidade, segurança, confiança, valorização.

O questionário foi avaliado, em uma primeira fase, por cinco especialistas (amostra não probabilística por julgamento) para aperfeiçoamento e adequação dele para que, em uma segunda fase, fosse realizado o pré-teste com 15 consumidores de carne - amostra não probabilística por conveniência - do instrumento de coleta de dados com o intuito de minimizar possíveis erros. A amostra respondeu à pesquisa por meio de uma survey via internet, sendo o questionário construído por meio do software Sphinx versão 5.0. O período de realização da coleta de dados foi do dia 10 de setembro ao dia 21 de novembro de 2008, totalizando 60 dias. As respostas obtidas foram analisadas pelo software citado anteriormente. A taxa de retorno dos questionários da pesquisa foi de $10 \%$, totalizando uma amostra composta por 272 consumidores de carne bovina de diversas cidades do Brasil. A amostra foi considerada do tipo não probabilística por conveniência.

\section{RESULTADOS E DISCUSSÃO}

Na primeira dimensão analisada, com relação ao perfil dos consumidores entrevistados, a amostra é composta, majoritariamente, por homens $63,1 \%$-, com idade entre 25 e 44 anos, 66,5\%, e com alta escolaridade - 75,3\% com graduação ou pós-graduação. A amostra apresentou-se com uma renda familiar considerada elevada, superior a 10 salários mínimos, $56,5 \%$ dos consumidores, em sua maioria com duas pessoas residindo por moradia, 34,2\% (Tab. 1). 
Tabela 1. Caracterização do consumidor de carne

\begin{tabular}{|c|c|c|}
\hline \multicolumn{2}{|l|}{ Caracterização do consumidor } & Frequência \\
\hline \multirow[t]{2}{*}{ Gênero } & Feminino & $36,9 \%$ \\
\hline & Masculino & $63,1 \%$ \\
\hline \multirow[t]{4}{*}{ Idade } & $<24$ anos & $14,3 \%$ \\
\hline & 25 a 44 anos & $66,5 \%$ \\
\hline & 45 a 64 anos & $19,2 \%$ \\
\hline & $>65$ anos & $0 \%$ \\
\hline \multirow[t]{7}{*}{ Escolaridade } & $1^{\circ}$ grau incompleto & $0 \%$ \\
\hline & $1^{\circ}$ grau completo & $0,4 \%$ \\
\hline & $2^{\circ}$ grau incompleto & $0,4 \%$ \\
\hline & $2^{\circ}$ grau completo & $1,8 \%$ \\
\hline & $3^{\circ}$ grau incompleto & $22,1 \%$ \\
\hline & $3^{\circ}$ grau completo & $25,7 \%$ \\
\hline & Pós-graduação & $49,6 \%$ \\
\hline \multirow[t]{4}{*}{ Renda familiar (salários mínimos) } & Até $3 \mathrm{SM}$ & $6,3 \%$ \\
\hline & 3 a $10 \mathrm{SM}$ & $37,2 \%$ \\
\hline & 10 a $20 \mathrm{SM}$ & $39,8 \%$ \\
\hline & Mais de $20 \mathrm{SM}$ & $16,7 \%$ \\
\hline \multirow[t]{6}{*}{ Atividade } & Do lar & $0,4 \%$ \\
\hline & Estudante & $33 \%$ \\
\hline & Profissional liberal & $18 \%$ \\
\hline & Funcionário público & $15,1 \%$ \\
\hline & Empresa particular & $23,9 \%$ \\
\hline & Outro & $9,6 \%$ \\
\hline \multirow[t]{6}{*}{ Pessoas na residência } & 1 & $6,0 \%$ \\
\hline & 2 & $34,2 \%$ \\
\hline & 3 & $27 \%$ \\
\hline & 4 & $24,4 \%$ \\
\hline & 5 & $5,3 \%$ \\
\hline & Mais de 5 & $3,1 \%$ \\
\hline
\end{tabular}

Ao analisar o comportamento de consumo e de compra de carnes, é possível inferir que a carne bovina é a mais consumida, $82 \%$. O perfil dos respondentes alcança um nicho peculiar de consumidores de carne, em que $84 \%$ dos respondentes consomem este produto diariamente (Tab. 2). Além disso, o consumo específico de carne bovina pode ser considerado alto, uma vez que $45 \%$ dos entrevistados a consomem diariamente. Dados semelhantes foram apresentados por Delgado et al. (2006), os quais identificaram que a carne de preferência dos consumidores era a bovina e que o seu consumo ocorria entre o diário e três vezes por semana.
Foi possível constatar que o local de compra preferido para carne bovina são os supermercados, $80 \%$ (Tab. 3). Ademais, a compra da carne bovina em supermercados teve um significativo aumento, comparando-se os dados dessa pesquisa com os resultados de Barcellos (2004), a qual identificou, em 2002, que $60 \%$ das compras eram realizadas nos supermercados. Já no presente trabalho, realizado no ano de 2008, $80 \%$ dos respondentes realizam as compras nesse tipo de estabelecimento. 
Tabela 2. Comportamento e consumo de carnes

\begin{tabular}{llc} 
Pergunta & Opção de resposta & Frequência \\
\hline \multirow{2}{*}{ Carne que mais consome } & Aves & $14 \%$ \\
& Bovina & $82 \%$ \\
& Ovina & $1 \%$ \\
& Peixes & $2 \%$ \\
& Suína & $1 \%$ \\
& Diariamente & $84 \%$ \\
Consumo de carne & 2 vezes por semana & $2 \%$ \\
& 3 vezes por semana & $11 \%$ \\
& 1 vez a cada 15 dias & $2 \%$ \\
& Não consumo carne & $1 \%$ \\
Consumo de carne bovina & Diariamente & $45 \%$ \\
& 2 vezes por semana & $10 \%$ \\
& 3 vezes por semana & $40 \%$ \\
\hline
\end{tabular}

Tabela 3. Processo de compra de carne bovina

\begin{tabular}{clc}
\hline Pergunta & Opção de resposta & Frequência \\
\hline \multirow{3}{*}{ Onde compra carne bovina } & Açougue & $13 \%$ \\
& Supermercado & $80 \%$ \\
& Mercado público & $2 \%$ \\
& Casa especializada em carnes & $1 \%$ \\
& Outro & $5 \%$ \\
& & $11 \%$ \\
Quando compra carne bovina & 1 vez/mês & $24 \%$ \\
& A cada 15 dias & $47 \%$ \\
& 1 vez/semana & $17 \%$ \\
& 2 a 3 vezes/semana & $2 \%$ \\
Compras no supermercado & Todos os dias & $58 \%$ \\
& & $42 \%$ \\
\hline
\end{tabular}

Essa preferência pelos supermercados pode ser devido à facilidade de compra de carne bovina, uma vez que a agilidade e a praticidade no momento da compra são itens, geralmente, reconhecidos pelo consumidor de carne bovina. Assim, a conveniência buscada pelos consumidores é maximizada nos supermercados, considerando-se que a maioria opta por realizar as compras de carne semanalmente, $47 \%$, especialmente nas gôndolas dos supermercados, $58 \%$, em detrimento dos açougues (Tab. 3 ).

Na segunda dimensão do questionário, foram avaliados aspectos específicos das IG em carnes, incluindo aspectos de consumo, percepção de qualidade, segurança, confiança e valorização deste atributo. Com relação ao consumo de carnes com IG, a maioria dos respondentes,
$65,4 \%$, não havia consumido tal produto. No entanto, dos 34,6\% que já haviam, 16,2\% consumiram mais de 10 vezes esse produto com apelação de origem (Tab. 4). Cabe salientar a importância da fidelidade do consumidor a esse produto com indicação de origem geográfica, pois os entrevistados tendem a repetir o processo da compra, uma vez que adquirem esse bem com certificação de origem. Tendo em vista esse comportamento de consumo de produtos com IG, uma possibilidade seria utilizar para promoção das carnes com tal diferenciação, campanhas fortes de marketing. Essa atitude poderia ser direcionada especialmente aos supermercados e às gôndolas, locais mais procurados pelos entrevistados para compra, incentivando que o consumidor conheça a IG e adquira esse produto pela primeira vez. 
Tabela 4. Consumo de produtos com IG

\begin{tabular}{llc}
\hline Pergunta & Opção de resposta & $\begin{array}{c}\text { F } \\
\text { Frequência }\end{array}$ \\
\hline Já consumiu produto com IG & Sim & $34,6 \%$ \\
& Não & $65,4 \%$ \\
Se sim, quantas vezes & 1 vez & $4,2 \%$ \\
& Até 5 vezes & $9,8 \%$ \\
& Até 10 vezes & $4,4 \%$ \\
& Mais de 10 vezes & $16,2 \%$ \\
\hline
\end{tabular}

$\mathrm{Na}$ etapa seguinte da pesquisa, foi analisada a relação entre qualidade da carne bovina e a indicação geográfica, uma vez que a certificação de origem pode influenciar a imagem que o consumidor faz de determinado produto, sendo capaz, portanto, de diferenciá-lo dos demais. A IG pode sinalizar qualidade e interferir na avaliação dos atributos intrínsecos em função da associação do conceito com a adoção de um controle de qualidade mais rigoroso e com maior experiência no processo produtivo, transmitindo uma imagem de produto tradicional e único (Fandos e Flavián, 2006).

Como resultado deste estudo, os consumidores consideraram que a IG indica qualidade para carne, $81 \%$ dos respondentes. Dessa forma, a certificação dos produtos alimentares por meio das IG pode se converter em uma importante ferramenta de comercialização, uma vez que esse atributo sinaliza qualidade.

A partir dos resultados, verificou-se que as carnes com IG oferecem mais segurança, $91,1 \%$, ou seja, o alimento é considerado mais seguro, podendo minimizar o risco para a saúde. $\mathrm{Da}$ mesma forma, a maioria dos consumidores demonstrou que a carne com IG é mais confiável, $79 \%$, do que a carne sem este atributo (Fig. 1). Isso mostra que, para os consumidores, o selo de indicação geográfica oferece credibilidade e pode representar uma imagem positiva ao produto.

Além da qualidade, aspectos referentes à segurança e à confiança são considerados pelos consumidores de carne no momento da compra (Jahn et al., 2005; Akatu, 2006). Durante o processo de compra, os consumidores percebem atributos diferenciados em produtos com indicação geográfica (Falcão e Révillion, 2010), sendo esta uma oportunidade de agregação de valor ao produto, de ampliação de mercados e de expansão para nichos específicos.

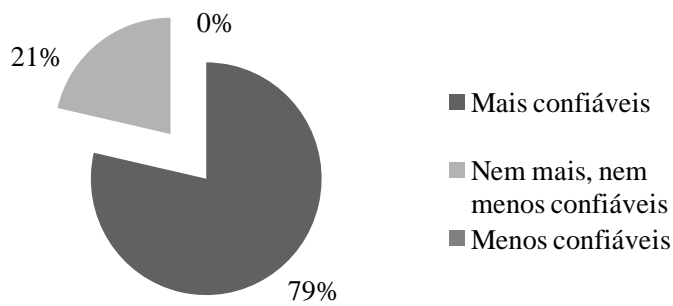

Figura 1. Confiança de carnes com indicação geográfica.

A partir da valorização dos atributos diferenciados da carne com IG percebidos pelo consumidor - qualidade, segurança e confiança -, verificou-se que os consumidores estão dispostos a pagar mais por esse selo. Nesse sentido, a maioria dos consumidores -aproximadamente $70 \%$ - está disposta a pagar mais pelo produto, sendo que $32,6 \%$ dos entrevistados pagariam até 5\% a mais pela indicação geográfica em carnes (Fig. 2).

Em um trabalho semelhante, Velho et al. (2009) observaram que os consumidores exigiram alimentos com qualidade e certificados confiáveis que demonstrem e garantam a qualidade dos alimentos. Contudo, estão dispostos a pagar somente um pouco a mais, 5 a $10 \%$, pela certificação, em comparação ao valor atual de mercado, podendo esta resposta estar associada ao fato de sua renda mensal não permitir maior valorização desse atributo. 


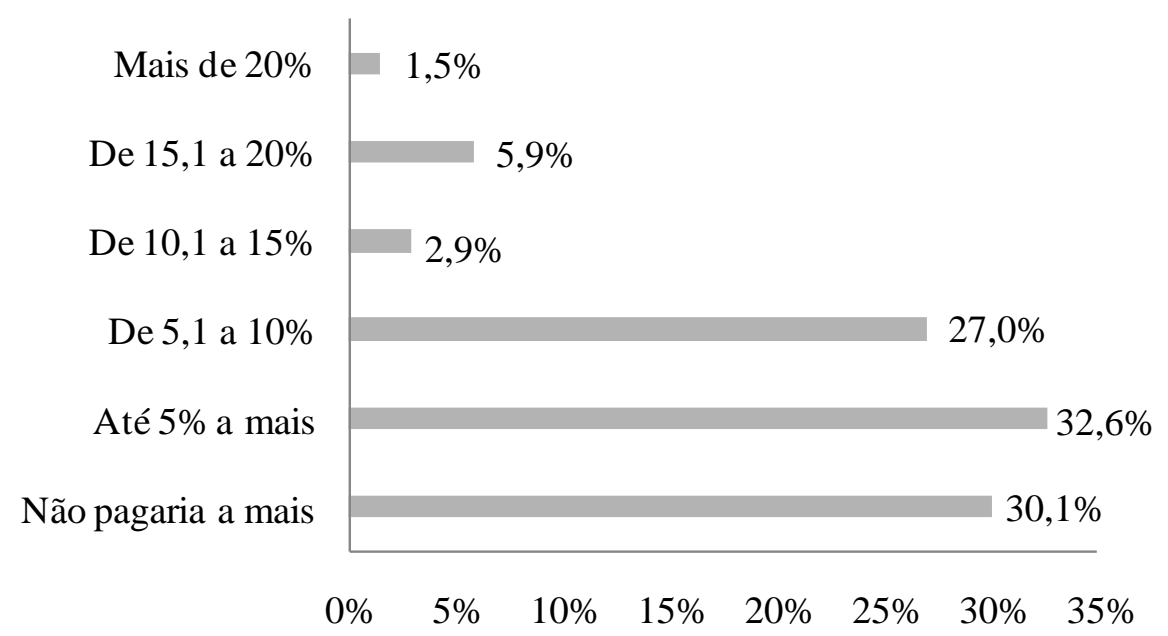

Figura 2. Disposição para pagar pela carne com indicação geográfica.

A valorização do consumidor em relação ao produto com IG pode influenciar positivamente essa certificação de origem em carnes, considerando-se que existe um nicho de mercado interessado no produto. Assim, identifica-se que a IG em carne pode viabilizar a agregação de valor no produto final, visto que os consumidores reconhecem atributos diferenciados neste produto - qualidade, segurança e confiança -, além de estarem dispostos a pagar mais por esses produtos.

\section{CONCLUSÕES}

Os consumidores reconhecem a indicação geográfica em carnes como um indicador de qualidade e mostram-se dispostos a pagar mais por essa diferenciação. Além de perceber qualidade, o consumidor considera que essa certificação oferece maior segurança e confiança ao produto. A partir desses atributos diferenciais, é possível direcionar este produto para nichos específicos de mercado e agregar valor à carne com IG.

\section{AGRADECIMENTOS}

À CAPES, agência financiadora da pesquisa.

\section{REFERENCIAS}

AKATU, Instituto. Você conhece os selos e etiquetas?, 2006. Disponível em:

<http://www.akatu.net>. Acessado em: 05 nov. 2010.

ASSAEL, H. Consumer behavior and marketing action. 4.ed. Boston: PWS-KENT Pub., 1992. 748p.

BARCELLOS, M.D. Informação e qualidade na compra de carne bovina. FACES R. Adm., v.3, p.4359, 2004.

DELGADO, E.F.; AGUIAR, A.P.; ORTEGA, E.M.M. et al. Brazilian consumers' perception of tenderness of beef steaks classified by shear force and taste. Sci. Agric., v.63, p.232-239, 2006.

FALCÃO, T.F.; RÉVILLION, J.P.P. A indicação geográfica de vinhos finos segundo a percepção de qualidade de enófilos. Cienc. Rural, v.40, p.453-458, 2010.

FANDOS, C.; FLAVIÁN, C. Intrinsic and extrinsic quality attributes, loyalty and buying intention: an analysis for PDO product. British Food J., v.108, p.646-662, 2006.

FONT I FURNOLS, M.; REALINI, C.; MONTOSSI, F. et al. Consumer's purchasing intention for lamb meat affected by country of origin, feeding system and meat price: A conjoint study in Spain, France and United Kingdom. Food Qual. Pref., v.22, p.443-451, 2011.

GUILHOTO, L.F.M. A influência do país de origem na percepção do consumidor sobre a qualidade dos produtos. Cad. Pesq. Adm., v.8, p.53-64, 2001. 
JAHN, G.; SCHRAMM, M.; SPILLER, A. The realiability of certification: quality labels as a consumer policy tool. J. Consum. Policy, v.28, p.5373,2005 .

MALAFAIA, G.C.; AZEVEDO, D.B. de; BARCELLOS, J.O.J. Terroir, empreendedorismo e mecanismos de coordenação na pecuária de corte. Rev. Bras. Zootec., v.40, supl. especial, p.195-203, 2011.

MENAPACE, L.; COLSON, G.; GREBITUS, C. et al. Consumers' preferences for geographical origin labels: evidence from the Canadian olive oil market. Eur. Rev. Agric. Econ., v.38, p.193-212, 2011.

NEMECEK; T.; HUGUENIN-ELIE, O.; DUBOIS, D. et al. Life cycle assessment of Swiss farming systems: II. Extensive and intensive production. J. Agric. Syst., v.104, p.233-45, 2011.

ROJAS, L.G.G. Indicaciones geograficas y denominaciones de origen. Un aporte para su implementaciónen. Costa Rica: ICAR-PRODARMAG-CNP, 2004. 160p.

SCHNETTLER, B.; MIRANDA, H.; SEPULVEDA, $\mathrm{J}$. et al. Importance of origin in the purchase of chicken meat in Central-Southern. Rev. Cient., v.21, p.317-226, 2011.

SEPÚLVEDA, W.; MAZA, M.T.; MANTECÓN, A.R. Factors that affect and motivate the purchase of quality-labelled beef in Spain. Meat Sci., v.80, p.12821289, 2008.
TÁPIAS, B.A. Indicação geográfica como instrumento de política de valorização de produtos agropecuários: o papel do Ministério da Agricultura, Pecuária e Abastecimento. In: LAGARES, L.; LAGES, V.; BRAGA, C. (Org.). Valorização de produtos com diferencial de qualidade e identidade: Indicações Geográficas e certificações para competitividade nos negócios. 2.ed. Brasília: SEBRAE, 2005. p.23-30.

VARGAS, I.C.S. Indicações geográficas no Brasil: possibilidades para os produtores inseridos na área de proteção ambiental do Ibirapuitã-RS. 2008. $116 \mathrm{f}$. Dissertação (Mestrado em Extensão Rural) Programa de Pós-Graduação em Extensão Rural Universidade Federal de Santa Maria, Santa Maria, RS.

VELHO, J.P.; BARCELLOS, J.O.J.; LENGLER, L. $e t$ al. Disposição dos consumidores porto-alegrenses à compra de carne bovina com certificação. Rev. Bras. Zootec., v.38, p.399-404, 2009.

ZUIN, L.F.S.; ZUIN, P.B. Produção de alimentos tradicionais: contribuindo para o desenvolvimento local/regional e dos pequenos produtores rurais. Rev. Bras. Gest. Desenvolv. Reg., v.4, p.109-127, 2008. 